\title{
MARKOV TYPE CONSTANTS, FLAT TORI AND WASSERSTEIN SPACES
}

\author{
VLADIMIR ZOLOTOV
}

\begin{abstract}
Let $M_{p}(X, T)$ denote the Markov type $p$ constant at time $T$ of a metric space $X$, where $p \geq 1$. We show that $M_{p}(Y, T) \leq M_{p}(X, T)$ in each of the following cases:

(a) $X$ and $Y$ are geodesic spaces and $Y$ is covered by $X$ via a finitesheeted locally isometric covering,

(b) $Y$ is the quotient of $X$ by a finite group of isometries,

(c) $Y$ is the $L^{p}$-Wasserstein space over $X$.

As an application of (a) we show that all compact flat manifolds have Markov type 2 with constant 1 . In particular the circle with its intrinsic metric has Markov type 2 with constant 1 . This answers the question raised by S.-I. Ohta and M. Pichot.

Parts (B) and (C) imply new upper bounds for Markov type constants of the $L^{p}$-Wasserstein space over $\mathbb{R}^{d}$. These bounds were conjectured by A. Andoni, A. Naor and O. Neiman. They imply certain restrictions on bi-Lipschitz embeddability of snowflakes into such Wasserstein spaces.
\end{abstract}

\section{INTRODUCTION}

Let $X$ be a metric space, $p \geq 1$, and $T \in \mathbb{N}$. We denote by $M_{p}(X, T) \in$ $[0, \infty)$ the Markov type $p$ constant at time $T$ of $X$, see Definition [2.3. The Markov type $p$ constant of $X$, denoted by $M_{p}(X)$ is defined by

$$
M_{p}(X)=\sup _{T \in \mathbb{N}} M_{p}(X, T) \in[1, \infty] .
$$

We say that $X$ has Markov type $p$ if $M_{p}(X)<\infty$.

Ball [2] introduced the concept of Markov type in his study of the Lipschitz extension problem. Major results in this direction were obtained later by Naor, Peres, Schramm and Sheffield [10. The notion of Markov type has also found applications in the theory of bi-Lipschitz embeddings [3,7].

It was shown in [13] that if $X$ is a geodesic metric space with $M_{2}(X)=1$ then $X$ is nonnegatively curved in sense of Alexandrov. Ohta [12] showed that there exists an universal constant $M_{A}$ such that every nonnegatively curved Alexandrov space $X$ has Markov type 2 with $M_{2}(X) \leq M_{A}$. The

2010 Mathematics Subject Classification. 51F99.

Key words and phrases. Markov type, Alexandrov space, Flat manifold, Wasserstein space. 
best known bound $M_{A} \leq \sqrt{1+\sqrt{2}+\sqrt{4 \sqrt{2}-1}}=2.08 \ldots$ is due to Andoni, Naor, and Neiman [1].

The authors of [1] mentioned that it is plausible that $M_{A}=1$. Thus we have the following question.

Question 1.1 (A. Andoni, A. Naor, O. Neiman [1]). Is it true that $M_{2}(X)=1$ for every Alexandrov space $X$ of nonnegative curvature?

In the original paper [2] (see also [7]) Ball shows that Hilbert spaces have Markov type 2 with constant 1 . Therefore $M_{2}(X)=1$ for every subset $X$ of $L^{2}$. Thus convex subsets of Hilbert spaces are examples of geodesic spaces with Markov type 2 constant 1 . But it seems that no other examples of geodesic spaces having Markov type 2 with constant 1 are known. The following two theorems allow us to expand the list of such examples. Though the theorems are motivated by Question 1.1, they do not involve Alexandrov geometry.

Theorem 1. Let $p \geq 1$, and let $X, Y$ be metric spaces such that at least one of the following holds:

(1) $X$ and $Y$ are geodesic spaces and $Y$ is covered by $X$ via a finitesheeted locally isometric covering,

(2) $Y$ is a quotient of $X$ by a finite group of isometries,

(3) $Y$ is the $L^{p}$-Wasserstein space over $X$.

Then for every $T \in \mathbb{N}$ we have $M_{p}(X, T) \geq M_{p}(Y, T)$ and $M_{p}(X) \geq M_{p}(Y)$.

Theorem 1 is a merger of propositions. See Proposition 4.2 for the case (11), Proposition 5.2 for the case (2) and Proposition 6.2 for the case (3).

Though the finiteness assumptions in Theorem[1(1, 2) may seem unnatural they can not be dropped, see Example 7.4.

Recall that a Riemannian manifold $(M, g)$ is called flat if it is locally isometric to the Euclidean space. As an application of Theorem 11(1) we show that compact flat manifolds have Markov type 2 with constant 1 .

Theorem 2. Compact flat Riemannian manifolds have Markov type 2 with constant 1 . In particular $M_{2}\left(S^{1}\right)=1$, where $S^{1}$ is a circle with its intrinsic metric.

This answers the question raised by S.-I. Ohta and M. Pichot [13], see also [1].

Let $X$ be an Alexandrov space of nonnegative curvature and $p=2$. In each of the cases dealt with in Theorem 1 the space $Y$ is also nonnegatively curved in the sense of Alexandrov (see [6] Section 4.6 for the cases (1), (2), and [14] Proposition 2.10.iv for the case (3) ). Hence we can consider Theorem 1 as a supporting evidence for the affirmative answer to Question 1.1. 
For a metric space $X$, we denote by $\mathscr{P}_{p}(X)$ the $p$-Wasserstein space over $X$, see Section 6. As a consequence of Theorem 1,(2,3) we obtain the following upper bounds on Markov type $p$ constants for the $p$-Wasserstein space over Euclidean space $\mathbb{R}^{d}$.

Corollary 3. For every $p \in(2, \infty)$ and $T, d \in \mathbb{N}$ we have

(1) $M_{p}\left(\mathscr{P}_{p}\left(\mathbb{R}^{d}\right), T\right) \leq 16 d^{\frac{1}{2}-\frac{1}{p}} p^{\frac{1}{2}} T^{\frac{1}{2}-\frac{1}{p}}$,

(2) $M_{2}\left(\mathscr{P}_{p}\left(\mathbb{R}^{d}\right)\right) \leq 4 d^{\frac{1}{2}-\frac{1}{p}} \sqrt{p-1}$.

The upper bound for Markov type 2 constant of $\mathscr{P}\left(\mathbb{R}^{d}\right)$ given by Corollary 3(2) imply certain extension theorem for partial Lipschitz maps from $\mathscr{P}\left(\mathbb{R}^{d}\right)$ into CAT(0) spaces, uniformly convex Banach spaces or more generally metric spaces with metric Markov cotype 2 (See [8, Theorem 1.11, Corollary $1.13])$.

For a metric space $\left(X, d_{X}\right)$ and $\alpha \in(0,1]$, the metric space $\left(X, d_{X}^{\alpha}\right)$ is called the $\alpha$-snowflake of $\left(X, d_{X}\right)$.

As observed by A. Andoni, A. Naor and O. Neiman (see [1, Section 3]) an upper bound for $M_{p}\left(\mathscr{P}_{p}\left(\mathbb{R}^{d}\right), T\right)$ implies certain restriction on the embeddability of snowflakes into $\mathscr{P}_{p}\left(\mathbb{R}^{d}\right)$. Namely we have the following corollary improving the estimate in [1, Theorem 2].

Corollary 4. For every $n>1$ there exists an $n$-point metric space $X_{n}$ such that for every $\alpha \in\left(\frac{1}{2}, 1\right]$, every $p \in(2, \infty)$ and every $d \in \mathbb{N}$ the $\alpha$-snowflake of $X_{n}$ does not admit an embedding to $\mathscr{P}_{p}\left(\mathbb{R}^{d}\right)$ with the bi-Lipschitz distortion less then $C d^{-\frac{1}{2}+\frac{1}{p}} p^{-\frac{1}{2}}(\log n)^{\alpha-\frac{1}{2}}$, where $C>0$ is an absolute constant.

Corollary 4 answers the question posed by A. Andoni, A. Naor and O. Neiman, see [1, Question 23].

Organization of the paper. Definitions, preliminaries and notation are discussed in Section 2. Lemmas for lifts of Markov chains are given in Section 3. Section 4 is devoted to results related to finite-sheeted locally isometric coverings. It contains the proof of Theorem 1(1) (see Proposition 4.2) and the proof of Theorem 2. Section 5 is an analogue of Section 4 for quotients by finite groups of isometries, it gives the proof of Theorem 1(2), see Proposition 5.2. In Section 6 we present results related to Wasserstein spaces and a proof of Theorem 1(3), see Proposition 6.2. In Section 7 we prove Corollaries 3 and 4. Section 7 also contains counter examples, a conjecture and some additional results about lifts of Markov chains. 
Acknowledgements. I thank my advisor Sergey V. Ivanov for all his ideas, advice and continuous support. I am grateful to Prof. Assaf Naor for valuable comments on the preliminary version of the paper which result in particular in Corollary 3(2). The paper is supported by the Russian Science Foundation under grant 16-11-10039.

\section{Definitions, PREliminaries And nOtation}

Let $\left\{Z_{t}\right\}_{t=0}^{\infty}$ be a Markov chain on a finite state space $S$ with transition probabilities $a_{i j}=\operatorname{Pr}\left[Z_{t+1}=j \mid Z_{t}=i\right], i, j \in S$. The Markov chain $\left\{Z_{t}\right\}_{t=0}^{\infty}$ is said to be stationary if $\pi_{i}=\operatorname{Pr}\left[Z_{t}=i\right]$ does not depend on $t=0,1,2, \ldots$ A stationary Markov chain $\left\{Z_{t}\right\}_{t=0}^{\infty}$ is said to be reversible if $\pi_{i} a_{i j}=\pi_{j} a_{j i}$, for every $i, j \in S$.

In order to construct a stationary reversible Markov chain on a finite set $S$, it suffices to define a nonnegative vector $\left(\pi_{i}\right)_{i \in S}$ and a nonnegative matrix $\left(a_{i j}\right)_{i, j \in S}$ and verify that

(2.1) vector $\pi$ is stochastic, i.e. $\sum_{i \in S} \pi_{i}=1$,

(2.2) matrix $a$ is stochastic, i.e $\sum_{j \in S} a_{i j}=1$, for every $i \in S$,

(2.3) $\pi_{i} a_{i j}=\pi_{j} a_{j i}$, for every $i, j \in S$.

The property (2],3) provides both stationarity and reversibility.

Recall that a sequence of random variables $W=\left\{W_{t}\right\}_{t=0}^{\infty}$ on a set $X$ is called a random walk.

Definition 2.1. We say that random walk $W$ on a set $X$ is a Markov walk if there exists a stationary reversible Markov chain $\left\{Z_{t}\right\}_{t=0}^{\infty}$ on a finite state space $S$ and a map $f: S \rightarrow X$ such that $W_{t}=f\left(Z_{t}\right)$.

We say that Markov walks $W$ and $\widetilde{W}$ on a set $X$ are equivalent if the probability measures on the space of sequences $X^{\infty}=\left\{\left\{x_{i}\right\}_{1}^{\infty} \mid x_{i} \in X\right\}$ induced by $W$ and $\widetilde{W}$ coincide.

Notation 2.2. Let $Z$ be a stationary reversible Markov chain on a finite state space $S$, and $S_{0}, \ldots, S_{T} \subset S$. We denote by $A^{Z}\left(S_{0}, \ldots, S_{T}\right)$ the probability $\operatorname{Pr}\left[Z_{0} \in S_{0}, \ldots, Z_{T} \in S_{T}\right]$. For $s \in S$ and $S_{1} \subset S$ we denote by $P^{Z}\left(s, S_{1}\right)$ the conditional probability $\operatorname{Pr}\left[Z_{1} \in S_{1} \mid Z_{0}=s\right]$.

For a Markov walk $W$ on a metric space $X$ and $T \in \mathbb{N}$, we denote by $\mathcal{E}_{p}(W, T)$ the expectation $\mathbb{E} d\left(W_{T}, W_{0}\right)^{p}$.

The following definition is a slightly reworded version of the one in $[1$, Section 3].

Definition 2.3. Let $X$ be a metric space, $T \in \mathbb{N}$ and $p \geq 1$. The Markov type $p$ constant at time $T$ of $X$, denoted by $M_{p}(X, T)$ is the infimum of all $K>0$ such that for every Markov walk $W$ on $X$,

$$
\mathcal{E}_{p}(W, T) \leq \underset{4}{K^{p}} T \mathcal{E}_{p}(W, 1)
$$


The Markov type $p$ constant $M_{p}(X)$ is defined by

$$
M_{p}(X)=\sup _{T \in \mathbb{N}} M_{p}(X, T) \in[1, \infty] .
$$

We say that $X$ has Markov type $p$ if $M_{p}(X)<\infty$.

For a metric space $X$ we denote by $\operatorname{diam}(X)$ the diameter of $X$, and by Iso $(X)$ the group of isometries of $X$.

Let $X$ and $Y$ be metric spaces, and $p \geq 1$. We write $X \times_{p} Y$ to denote the $p$-product space, i.e., the space with the distance defined by formula

$$
d\left(\left(x_{1}, y_{1}\right),\left(x_{2}, y_{2}\right)\right)^{p}=d_{X}\left(x_{1}, x_{2}\right)^{p}+d_{Y}\left(y_{1}, y_{2}\right)^{p} .
$$

We write $X_{p}^{n}$ to denote the $n$th $p$-power of $X$, i.e.,

$$
\left.X_{p}^{n}=X \times_{p} X \times_{p} \cdots \times_{p} X \text { ( } n \text { times }\right) .
$$

The symmetric group $S_{n}$ acts on $X_{p}^{n}$ by permutation of coordinates. We denote by $X_{p}^{n} / S_{n}$ the corresponding quotient metric space.

For $c>0$ we write $c X$ to denote the space with scaled metric, where the distance is defined by formula

$$
d_{c X}(x, y)=c d_{X}(x, y) .
$$

The following proposition immediately follows from the definitions

Proposition 2.4. For $X, Y$ metric spaces, $p \geq 1, c>0, n \in \mathbb{N}$ and $T \in \mathbb{N}$ we have

(1) $M_{p}\left(X \times_{p} Y, T\right)=\max \left\{M_{p}(X, T), M_{p}(Y, T)\right\}$,

(2) $M_{p}\left(X_{p}^{n}, T\right)=M_{p}(X, T)$,

(3) $M_{p}(c X, T)=M_{p}(X, T)$.

Let $U, V$ be two real-valued random variables. We write $U={ }_{s t} V$, if $U$ and $V$ are equal in distribution. For $U$ and $V$ defined on one probability space we write $U \stackrel{\text { a.s. }}{\leq} V$ if $U \leq V$ almost surely, i.e $\operatorname{Pr}[U>V]=0$.

Definition 2.5. Let $X$ and $Y$ be two sets and let $\chi: X \rightarrow Y$ be a map. Let $\widetilde{W}$ and $W$ be Markov walks on $X$ and $Y$. We say that $\widetilde{W}$ is a lift of $W$ along $\chi$, if Markov walks $\chi(\widetilde{W})$ and $W$ are equivalent, see Definition 2.1.

In the case when $X$ and $Y$ are metric spaces and in addition to the previous property we have $d\left(\widetilde{W}_{1}, \widetilde{W}_{0}\right)={ }_{s t} d\left(W_{1}, W_{0}\right)$, we say that $\widetilde{W}$ is a metric lift of $W$ along $\chi$.

Proposition 2.6. Let $X, Y$ be metric spaces and $\chi: X \rightarrow Y$ a short map. Suppose that $\widetilde{W}$ is a metric lift of $W$ along $\chi$ then

(1) $d\left(\widetilde{W}_{1}, \widetilde{W}_{0}\right) \stackrel{a . s .}{\leq} \operatorname{diam}(Y)$,

(2) $\frac{\mathcal{E}_{p}(W, T)}{T \mathcal{E}_{p}(W, 1)} \leq \frac{\mathcal{E}_{p}(\widetilde{W}, T)}{\left.T \mathcal{E}_{p} \widetilde{W}, 1\right)}$, for every $T \geq 2$ and every $p \geq 1$. 
Proof. The first claim follows from the definition of a metric lift. The definition of a metric lift also implies that,

$$
\mathcal{E}_{p}(\widetilde{W}, 1)=\mathcal{E}_{p}(W, 1) \text {, for every } p \geq 1 .
$$

From the definition of a lift and the fact that $\chi$ is a short map we have,

$$
\mathcal{E}_{p}(\widetilde{W}, T) \geq \mathcal{E}_{p}(W, T) \text {, for every } T \geq 2 \text { and every } p \geq 1
$$

Thus implies the second claim.

The plan of the proof of Theorem 1(1) is to show that every Markov walk on the base space can be lifted to the covering space and apply Proposition 2.6(2) to the lift.

Definition 2.7. For a stationary reversible Markov chain $\left\{\widetilde{Z}_{t}\right\}_{t=0}^{\infty}$ on $\widetilde{S}$ we say that $\widetilde{Z}$ is restricted by $E \subset \widetilde{S} \times \widetilde{S}$ if $A^{\widetilde{Z}}(\{x\},\{y\})=0$, for every $x, y \in \widetilde{S}$ such that $(x, y) \notin E$, see Notation 2.2 .

Let $S, \widetilde{S}$ be finite sets, $E \subset \widetilde{S} \times \widetilde{S}$ be a symmetric subset and $\sigma: \widetilde{S} \rightarrow S$ be a map. For $x \in \widetilde{S}$ and $V \subset \widetilde{S}$ we denote by $\operatorname{deg}_{E}(x, V)$ the number of elements of $\{y \in V:(x, y) \in E\}$. The following definition provides a condition on $E$ which implies that every stationary reversible Markov chain on $S$ admits a lift along $\sigma$ restricted by $E$, see Lemma 3.2.

Definition 2.8. We say that $\sigma$ is regular with respect to $E$ if $\operatorname{deg}_{E}\left(x, \sigma^{-1}(s)\right)=$ $\operatorname{deg}_{E}\left(y, \sigma^{-1}(s)\right) \neq 0$, for every $s \in S$ and every $x, y \in \widetilde{S}$ such that $\sigma(x)=$ $\sigma(y)$.

\section{Lifts OF MARKOV CHAINS}

The next lemma provides a sufficient condition for being a lift of a Markov chain. A more complicated argument shows that this condition is also necessary, see Lemma [7.9.

Lemma 3.1. Let $\left\{Z_{t}\right\}_{t=0}^{\infty}$ and $\left\{\widetilde{Z}_{t}\right\}_{t=0}^{\infty}$ be stationary reversible Markov chains on finite sets $S$ and $\widetilde{S}$ and $\sigma: \widetilde{S} \rightarrow S$ a map such that,

(1) $A^{\widetilde{Z}}\left(\sigma^{-1}(s)\right)=A^{Z}(\{s\})$, for every $s \in S$,

(2) $P^{\widetilde{Z}}\left(\widetilde{s}_{1}, \sigma^{-1}\left(s_{2}\right)\right)=P^{Z}\left(\sigma\left(\widetilde{s}_{1}\right),\left\{s_{2}\right\}\right)$, for every $\widetilde{s}_{1} \in \widetilde{S}, s_{2} \in S$.

Then $\widetilde{Z}$ is a lift of $Z$ along $\sigma$.

Proof. We have to show that for every $T \in \mathbb{N}$ and every $s_{0}, \ldots, s_{T} \in S$,

$$
A^{\widetilde{Z}}\left(\sigma^{-1}\left(s_{0}\right), \sigma^{-1}\left(s_{1}\right), \ldots, \sigma^{-1}\left(s_{T}\right)\right)=A^{Z}\left(\left\{s_{0}\right\},\left\{s_{1}\right\}, \ldots,\left\{s_{T}\right\}\right) .
$$

The property (11) provides the case $T=0$. The general case follows from (2) by induction. 
The following lemma is a main technical tool of the paper.

Lemma 3.2. Let $S, \widetilde{S}$ be finite sets, $\sigma: \widetilde{S} \rightarrow S$ a regular map with respect to a symmetric set $E \subset \widetilde{S} \times \widetilde{S}$ and $\left\{Z_{t}\right\}_{t=0}^{\infty}$ a stationary reversible Markov chain on $S$. Then there exists a stationary reversible Markov chain $\left\{\widetilde{Z}_{t}\right\}_{t=0}^{\infty}$ on $\widetilde{S}$ such that $\widetilde{Z}$ is a lift of $Z$ along $\sigma$ and $\widetilde{Z}$ is restricted by $E$.

Proof. Let $\pi_{x}, a_{x y}$ be the stationary distribution and the transition matrix for $Z_{t}$. For $x \in \widetilde{S}$ we denote $\sigma^{-1}(\sigma(x))$ by $M_{x}$. We define a Markov chain $\widetilde{Z}$ by a distribution $\widetilde{\pi}_{x}=\frac{\pi_{\sigma(x)}}{\left|M_{x}\right|}$ and a transition matrix

$$
\widetilde{a}_{x y}=\left\{\begin{array}{l}
\frac{a_{\sigma(x) \sigma(y)}}{\operatorname{deg}_{E}\left(x, M_{y}\right)},(x, y) \in E, \\
0,(x, y) \notin E .
\end{array}\right.
$$

First we are going to show that $\widetilde{\pi}_{x}, \widetilde{a}_{x y}$ correctly define a stationary reversible Markov chain, i.e. to check the properties (2) 11)-(2],3). Properties (2]10), (2]2) and the case $(x, y) \notin E$ of (2) [3) follows directly from the definitions of $\widetilde{\pi}$ and $\widetilde{a}$.

In order to verify the case $(x, y) \in E$ of (2, 3) we have to show that $\widetilde{\pi}_{x} \widetilde{a}_{x y}=$ $\widetilde{\pi}_{y} \widetilde{a}_{y x}$ for every $x, y \in \widetilde{S}$ such that $(x, y) \in E$. Fix $x, y \in \widetilde{S}$, let $N$ be the number of elements of the set $\left(M_{x} \times M_{y}\right) \cap E$. Since $\sigma$ is regular with respect to $E$ we have

$$
\left|M_{x}\right| \operatorname{deg}_{E}\left(x, M_{y}\right)=N=\left|M_{y}\right| \operatorname{deg}_{E}\left(y, M_{x}\right)
$$

Thus,

$$
\begin{aligned}
& \widetilde{\pi}_{x} \widetilde{a}_{x y}=\frac{\pi_{\sigma(x)}}{\left|M_{x}\right|} \frac{a_{\sigma(x) \sigma(y)}}{\operatorname{deg}_{E}\left(x, M_{y}\right)}=\frac{\pi_{\sigma(x)} a_{\sigma(x) \sigma(y)}}{N}= \\
& =\frac{\pi_{\sigma(y)} a_{\sigma(y) \sigma(x)}}{N}=\frac{\pi_{\sigma(y)}}{\left|M_{y}\right|} \frac{a_{\sigma(y) \sigma(x)}}{\operatorname{deg}_{E}\left(y, M_{x}\right)}=\widetilde{\pi}_{y} \widetilde{a}_{y x} .
\end{aligned}
$$

As a result we have defined a stationary reversible Markov chain $\widetilde{Z}$.

Secondly, we have to show that $\widetilde{Z}$ is a lift of $Z$ along $\sigma$. From the definition of $\widetilde{\pi}$ we have

$$
A^{\widetilde{Z}}\left(\sigma^{-1}(s)\right)=\pi_{s}, \text { for every } s \in S .
$$

and the definition of $\widetilde{a}$ provides

$$
P^{\widetilde{Z}}\left(x, \sigma^{-1}(s)\right)=a_{\sigma(x) s}, \text { for every } x \in \widetilde{S}, s \in S .
$$

Applying Lemma 3.1 we obtain the claim. 


\section{Coverings and proof of Theorem 2}

The following lemma implies Theorem 1(1), see Proposition 4.2.

Lemma 4.1. Let $X, Y$ be geodesic spaces and $\chi: X \rightarrow Y$ a $k$-sheeted locally isometric covering. Then every Markov walk on $Y$ admits a metric lift along $\chi$ (see Definition 2.5).

Proof. Let $W$ be a Markov walk on on $Y$ given by $W_{t}=f\left(Z_{t}\right)$, where $\left\{Z_{t}\right\}_{t=0}^{\infty}$ is a stationary reversible Markov chain on a finite set $S$ and $f$ is a map from $S$ to $Y$.

Define $\widetilde{S}=\{(s, x) \in S \times X: \chi(x)=f(s)\}$. We denote the projections from $\widetilde{S}$ to $S$ and $X$ by $\sigma$ and $\widetilde{f}$. For each unordered pair $\left\{s_{1}, s_{2}\right\}$ of (not necessary different) elements of $S$ fix a minimizing geodesic $\gamma_{s_{1} s_{2}}$ connecting $f\left(s_{1}\right)$ and $f\left(s_{2}\right)$. Let $E$ be a set of all pairs $\left(x_{1}, x_{2}\right) \in \widetilde{S} \times \widetilde{S}$ such that there exists a lift of $\gamma_{\sigma\left(x_{1}\right) \sigma\left(x_{2}\right)}$ connecting $\widetilde{f}\left(x_{1}\right)$ and $\widetilde{f}\left(x_{2}\right)$. Note that for every $\left(x_{1}, x_{2}\right) \in E$,

$$
d_{X}\left(\tilde{f}\left(x_{1}\right), \tilde{f}\left(x_{2}\right)\right)=d_{Y}\left(f\left(\sigma\left(x_{1}\right)\right), f\left(\sigma\left(x_{2}\right)\right)\right) .
$$

The existence and uniqueness of covering paths implies that $\operatorname{deg}_{E}\left(x, \sigma^{-1}(s)\right)=1$, for every $x \in \widetilde{S}, s \in S$. Hence $\sigma$ is a regular map with respect to $E$. Lemma 3.2 provides the existence of a stationary reversible Markov chain $\widetilde{Z}_{t}$ on $\widetilde{S}$, such that

(1) $\widetilde{Z}_{t}$ is a lift of $Z_{t}$ along $\sigma$,

(2) $\widetilde{Z}$ is restricted by $E$ (see Definition 2.7).

We define $\widetilde{W}$ by $\widetilde{W}_{t}=\widetilde{f}\left(\widetilde{Z}_{t}\right)$. The definitions of $\widetilde{S}$ and $\widetilde{f}$ imply that $\chi \circ \tilde{f}=f \circ \sigma$. Hence, the equivalence of $\sigma(\widetilde{Z})$ and $Z_{t}$ implies the equivalence of $\chi(\widetilde{W})$ and $W$. Finally $\widetilde{W}$ is a metric lift of $W$, which follows from properties (1), (2) and (4.1).

Proposition 4.2. Let $X, Y$ be geodesic spaces. Let $\chi: X \rightarrow Y$ be a finite sheeted locally isometric covering. Then for every $p \geq 1$ and $T \in \mathbb{N}$ we have $M_{p}(X, T) \geq M_{p}(Y, T)$ and $M_{p}(X) \geq M_{p}(Y)$.

Proof. The statement follows from Lemma 4.1] and Proposition 2.6.

Proof of Theorem 2. Let $X$ be a compact flat Riemannian manifold. By Bieberbach's Theorem [4,5], $X$ can be covered by a flat torus. Thus by Proposition 4.2 it suffices to consider the case $X=\mathcal{T}^{d}$, where $\mathcal{T}^{d}$ is a flat torus of dimension $d$.

Let $W$ be a Markov walk on $\mathcal{T}^{d}$. For every positive integer $k$ the flat torus $\mathcal{T}^{d}$ admits a locally isometric $k^{d}$-sheeted covering by the scaled torus $k \mathcal{T}^{d}$. Indeed, if $\mathcal{T}^{d}=\mathbb{R}^{d} / \Gamma$ where $\Gamma$ is a lattice, then $k \mathcal{T}^{d}=\mathbb{R}^{d} / k \Gamma$. The natural quotient map $\mathbb{R}^{d} / k \Gamma \rightarrow \mathbb{R}^{d} / \Gamma$ is a desired covering map. 
By Lemma 4.1, Proposition 2.6 and rescaling there exists a Markov walk $W^{k}$ on $\mathcal{T}^{d}$ such that

$$
\begin{aligned}
& d\left(W_{1}^{k}, W_{0}^{k}\right) \stackrel{\text { a.s. }}{\leq} \frac{\operatorname{diam}\left(\mathcal{T}^{d}\right)}{k} \\
& \frac{\mathcal{E}_{2}(W, T)}{T \mathcal{E}_{2}(W, 1)} \leq \frac{\mathcal{E}_{2}\left(W^{k}, T\right)}{T \mathcal{E}_{2}\left(W^{k}, 1\right)} .
\end{aligned}
$$

By the Nash embedding theorem (see [11]) there exists a (Riemannian) isometric $C^{1}$-map $\Phi: \mathcal{T}^{d} \rightarrow \mathbb{R}^{2 d}$. Then for every $\epsilon>0$ there exists $\delta(\epsilon)>0$ such that

$$
d(x, y) \leq(1+\epsilon)\|\Phi(x)-\Phi(y)\|,
$$

for every pair of points $x, y \in \mathcal{T}^{d}$ with $d(x, y)<\delta(\epsilon)$.

Fix $\epsilon>0$ and $T \in \mathbb{N}$. Choose $k>\frac{\operatorname{diam}\left(T^{d}\right) T}{\delta(\epsilon)}$. Then $d\left(W_{1}^{k}, W_{0}^{k}\right)<\delta(\epsilon) / T$. Hence,

$$
\mathcal{E}_{2}\left(W^{k}, T\right) \stackrel{\text { 4.4 }}{\leq}(1+\epsilon)^{2} \mathcal{E}_{2}\left(\Phi \circ W^{k}, T\right) \leq(1+\epsilon)^{2} T \mathcal{E}_{2}\left(\Phi \circ W^{k}, 1\right) \leq(1+\epsilon)^{2} T \mathcal{E}_{2}\left(W^{k}, 1\right)
$$

where the second inequality follows from $M_{2}\left(\mathbb{R}^{2 d}\right)=1$. Thus,

$$
\frac{\mathcal{E}_{2}(W, T)}{T \mathcal{E}_{2}(W, 1)} \stackrel{4.3}{\leq} \frac{\mathcal{E}_{2}\left(W^{k}, T\right)}{T \mathcal{E}_{2}\left(W^{k}, 1\right)} \stackrel{4.5}{\leq}(1+\epsilon)^{2}
$$

Since $\epsilon$ is arbitrary, it follows that $\mathcal{E}_{2}(W, T) \leq T \mathcal{E}_{2}(W, 1)$. Thus $M_{2}\left(\mathcal{T}^{d}\right)=1$ and Theorem 2 follows.

\section{Quotients BY Finite GRoups}

Recall that, a finite group $G$ acting by isometries on a metric space $X$ induces a quotient metric on $X / G$, given by $d_{X / G}(\bar{x}, \bar{y})=\min _{x \in \bar{x}, y \in \bar{y}} d_{X}(x, y)$. The following lemma is an analogue of Lemma 4.1 for quotient maps.

Lemma 5.1. Let $X$ be a metric space. Let $G$ be a finite subgroup of Iso( $X)$, and let $\chi: X \rightarrow X / G$ be the corresponding quotient map. Then every Markov walk on $X / G$ admits a metric lift along $\chi$.

Proof. The proof is similar to that of Lemma 4.1, the only difference is the construction of the set $E$. Let $W_{t}$ be a Markov walk on on $X / G$ given by $W_{t}=f\left(Z_{t}\right)$, where $\left\{Z_{t}\right\}_{t=0}^{\infty}$ is a stationary reversible Markov chain on a finite set $S$ and $f$ is a map from $S$ to $X / G$.

Define $\widetilde{S}=\{(s, x) \in S \times X: \chi(x)=f(s)\}$. We denote the projections from $\widetilde{S}$ to $S$ and $X$ by $\sigma$ and $\widetilde{f}$. Let $E$ be a set of all pairs $\left(x_{1}, x_{2}\right) \in \widetilde{S} \times \widetilde{S}$ such that $d_{X}\left(\tilde{f}\left(x_{1}\right), \tilde{f}\left(x_{2}\right)\right)=d_{X / G}\left(\underset{9}{f}\left(\sigma\left(x_{1}\right)\right), f\left(\sigma\left(x_{2}\right)\right)\right)$. 
Let $s_{1}, s_{2} \in S$ and $x_{1}, x_{2} \in \sigma^{-1}\left(s_{1}\right)$. Since $\sigma^{-1}\left(s_{1}\right)$ and $\sigma^{-1}\left(s_{2}\right)$ are orbits of an isometric action of a finite group, we have $\operatorname{deg}_{E}\left(x_{1}, \sigma^{-1}\left(s_{2}\right)\right)=\operatorname{deg}_{E}\left(x_{2}, \sigma^{-1}\left(s_{2}\right)\right) \neq 0$. Hence $\sigma$ is a regular map with respect to $E$.

The rest of the proof is the same as in Lemma 4.1.

Proposition 5.2. Let $X$ be a metric space and $G$ be a finite subgroup of Iso $(X)$. Then for every $p \geq 1$ and every $T \in \mathbb{N}$ we have $M_{p}(X, T) \geq$ $M_{p}(X / G, T)$ and $M_{p}(X) \geq M_{p}(X / G)$.

Proof. The statement follows from Lemma 5.1 and Proposition 2.6 .

\section{WASSERSTEIN SPACES}

For reader's convenience we recall the definition of Wasserstein spaces. For further details see [15].

Let $X$ be a metric space. Let $p \geq 1$ and let $\mu, \nu$ be Borel probabalistic measures with finite $p$-th moment, i.e

$$
\int_{X} d^{p}(x, o) d \mu(x)<\infty, \int_{X} d^{p}(x, o) d \nu(x)<\infty,
$$

for some (hence all) $o \in X$. We say that measure $q$ on $X \times X$ is a coupling of $\mu$ and $\nu$ iff its marginals are $\mu$ and $\nu$, that is, iff

$$
q(A \times X)=\mu(A), q(X \times A)=\nu(A),
$$

for all Borel measurable subsets $A \subset X$. The $L^{p}$-Wasserstein distance between $\mu$ and $\nu$ is defined by

$$
d_{W_{p}}(\mu, \nu)=\inf \left\{\left(\int_{X \times X} d^{p}(x, y) d q(x, y)\right)^{\frac{1}{p}}: q \text { is a coupling of } \mu \text { and } \nu\right\} .
$$

The $L^{p}$-Wasserstein space $\mathscr{P}_{p}(X)$ is the set of Borel probabilistic measures with finite $p$-th moment on $X$ equipped with $L^{p}$-Wasserstein distance.

Recall that, for a metric space $X$ we denote by $X_{p}^{n}$ the $p$-power of $X$ and by $X_{p}^{n} / S_{n}$ the quotient space of $X_{p}^{n}$ by permutations of coordinates. The following lemma allows to deduce Theorem 1(3) from Proposition [5.2, see Proposition 6.2.

Lemma 6.1. Let $X$ be a metric space, $n \in \mathbb{Z}$ and $p \geq 1$. The map $\Phi_{n}: n^{-\frac{1}{p}}\left(X_{p}^{n} / S_{n}\right) \rightarrow \mathscr{P}_{p}(X)$ defined by

$$
\Phi_{n}\left(x_{1}, \ldots, x_{n}\right)=\frac{1}{n} \delta\left(x_{1}\right)+\cdots+\frac{1}{n} \delta\left(x_{n}\right)
$$

is a distance preserving map. 
Proof. We denote $n^{-\frac{1}{p}}\left(X_{p}^{n} / S_{n}\right)$ by $Y$. Fix two points $w=\left(w_{1}, \ldots, w_{n}\right)$ and $q=\left(q_{1}, \ldots, q_{n}\right)$ in $Y$. The distance between $w$ and $q$ is given by

$$
d_{Y}^{p}(w, q)=\frac{1}{n} \inf _{s \in S_{n}} \sum_{i=1}^{n} d^{p}\left(w_{i}, q_{s(i)}\right) .
$$

Let $\mathcal{P}_{n}$ denote the set of all $n \times n$ permutation matrices and $D$ the $n \times n$ matrix defined by $D_{i j}=d^{p}\left(w_{i}, q_{j}\right)$. The formula for the distance can be rewritten as

$$
d_{Y}^{p}(w, q)=\frac{1}{n} \inf _{A \in \mathcal{P}_{n}} D \circ A,
$$

where $\circ$ denotes the Hadamard product (entrywise product) of matrices.

Let $\mathcal{D}$ denotes the set of all $n \times n$ doubly stochastic matrices. Then the Wasserstein distance between $\Phi_{n}(w)$ and $\Phi_{n}(q)$ can be written as

$$
d_{W_{p}}^{p}\left(\Phi_{n}(w), \Phi_{n}(q)\right)=\frac{1}{n} \inf _{A \in \mathcal{D}} D \circ A .
$$

By the Birkhoff-von Neumann theorem $\mathcal{D}$ is the convex hull of $\mathcal{P}_{n}$. Since the " $D \circ "$ is a linear functional it follows that

$$
\frac{1}{n} \inf _{A \in \mathcal{D}} D \circ A=\frac{1}{n} \inf _{A \in \mathcal{P}_{n}} D \circ A .
$$

Thus $d_{Y}(w, q)=d_{W_{p}}(\Phi(w), \Phi(q))$.

Proposition 6.2. Let $X$ be a a metric space, $p \geq 1$ and $T \in \mathbb{N}$. Then $M_{p}\left(\mathscr{P}_{p}(X), T\right)=M_{p}(X, T)$ and $M_{p}\left(\mathscr{P}_{p}(X)\right)=M_{p}(X)$.

Proof. For $k \in \mathbb{N}$ we denote by $I_{k}$ the image of $\Phi_{2^{k}}$, where $\Phi_{2^{k}}$ is the map defined in Lemma 6.1. Note that $I_{k} \subset I_{k+1}$ for every $k \in \mathbb{N}$. Since the union $\cup_{k=1}^{\infty} I_{k}$ is dense in $\mathscr{P}_{p}(X)$ (see [15]) we have

$$
M_{p}\left(\mathscr{P}_{p}(X), T\right)=\sup _{k \in \mathbb{N}} M_{p}\left(I_{k}, T\right) .
$$

From Lemma 6.1, Proposition 5.2 and Proposition 2.4 it follows that

$$
M_{p}\left(I_{k}, T\right)=M_{p}\left(\left(2^{k}\right)^{-\frac{1}{p}}\left(X_{p}^{\left(2 /^{k}\right)} / S_{2^{k}}\right), T\right) \leq M_{p}(X, T) .
$$

Hence we have $M_{p}\left(\mathscr{P}_{p}(X), T\right) \leq M_{p}(X, T)$. The existence of isometric copy of $X$ in $\mathscr{P}_{p}(X)$ implies the opposite inequality.

\section{Proofs of Corollaries 3 and 4 and Counter examples.}

Proof of Corollary 3(1). For $p>2$ and $T \in \mathbb{N}$ we have the following upper bound for $M_{p}(\mathbb{R}, T)$,

$$
M_{p}(\mathbb{R}, T) \leq 16 p^{\frac{1}{2}} T^{\frac{1}{2}-\frac{1}{p}}
$$


see [10, Theorem 4.5]. Proposition 2.4 implies that

$$
M_{p}\left(\mathbb{R}_{p}^{d}, T\right) \leq 16 p^{\frac{1}{2}} T^{\frac{1}{2}-\frac{1}{p}} .
$$

Since the $l_{p}$ norm on $\mathbb{R}^{d}$ is $d^{\frac{1}{2}-\frac{1}{p}}$-equivalent to the $l_{2}$ norm on $\mathbb{R}^{d}$, we obtain

$$
M_{p}\left(\mathbb{R}^{d}, T\right) \leq 16 d^{\frac{1}{2}-\frac{1}{p}} p^{\frac{1}{2}} T^{\frac{1}{2}-\frac{1}{p}} .
$$

Finally Proposition 6.2 provides an upper bound for $M_{p}\left(\mathscr{P}_{p}\left(\mathbb{R}^{d}\right), T\right)$,

$$
M_{p}\left(\mathscr{P}_{p}\left(\mathbb{R}^{d}\right), T\right) \leq 16 d^{\frac{1}{2}-\frac{1}{p}} p^{\frac{1}{2}} T^{\frac{1}{2}-\frac{1}{p}} .
$$

Definition 7.1 (see [1]). Let $X$ and $Y$ be metric spaces and $D \in[1, \infty]$. A mapping $f: X \rightarrow Y$ is said to have distortion at most $D$, if there exists $s \in(0, \infty)$ such that every $x, y \in X$ satisfy

$$
s d_{X}(x, y) \leq d_{Y}(f(x), f(y)) \leq D s d_{X}(x, y) .
$$

The infimum over those $D \in[1, \infty]$ for which this holds true is called the distortion of $f$ and is denoted by $\operatorname{dist}(f)$. The infimum of $\operatorname{dist}(f)$ over all $f: X \rightarrow Y$ is denoted by $c_{Y}\left(X, d_{X}\right)$.

We remind the reader that for a metric space $\left(X, d_{X}\right)$ and $\alpha \in(0,1]$, the metric space $\left(X, d_{X}^{\alpha}\right)$ is called the $\alpha$-snowflake of $\left(X, d_{X}\right)$.

Proof of Corollary 4. The following lemma provides a restriction on bi-Lipschitz embeddability of snowflakes into spaces with bounded Markov type constants.

Lemma 7.2 ( [1], Lemma 16). Fix a metric space $Y, T \in \mathbb{N}, K, p \in[1, \infty)$ and $\zeta \in[0,1]$. Suppose that

$$
M_{p}(Y, T) \leq K T^{\frac{\zeta(p-1)}{p}} .
$$

Denote $n=2^{4 T}$. Then trere exists an $n$-point metric space $\left(X, d_{X}\right)$ such that

$$
c_{Y}\left(X, d_{X}^{\alpha}\right) \geq C \frac{1}{K}(\log n)^{\alpha-\frac{1+\zeta(p-1)}{p}}, \text { for every } \alpha \in\left[\frac{1+\zeta(p-1)}{p}, 1\right],
$$

where $C>0$ is an absolute constant.

Lemma 7.2 as stated does not claim that $\left(X, d_{X}\right)$ do not depend on $p$, but the proof is given for $4 T$-dimensional discrete Hamming cube, i.e., $\left(X, d_{X}\right)=\left(\{0,1\}^{4 T},\|\cdot\|_{1}\right)$. Applying Lemma 7.2 to $Y=\mathscr{P}_{p}\left(\mathbb{R}^{d}\right), \zeta=\frac{\frac{p}{2}-1}{p-1}$ and $K=16 d^{\frac{1}{2}-\frac{1}{p}} p^{\frac{1}{2}}$ we obtain Corollary 4 .

Proof of Corollary 3(2). The proof is based on the following proposition.

Proposition 7.3 ( [10], Theorem 1.2). For $p \geq 2$ we have $M_{2}\left(L_{p}\right) \leq$ $4 \sqrt{p-1}$. 
Euclidean space $\mathbb{R}^{d}$ is $d^{\frac{1}{2}-\frac{1}{p}}$-equivalent to $\mathbb{R}_{p}^{d}$. Hence, $\mathscr{P}_{p}\left(\mathbb{R}^{d}\right)$ is $d^{\frac{1}{2}-\frac{1}{p}}$ equivalent to $\mathscr{P}_{p}\left(\mathbb{R}_{p}^{d}\right)$. Thus, $M_{2}\left(\mathscr{P}_{p}\left(\mathbb{R}^{d}\right)\right) \leq d^{\frac{1}{2}-\frac{1}{p}} M_{2}\left(\mathscr{P}_{p}\left(\mathbb{R}_{p}^{d}\right)\right)$.

The remaining part of the proof is similar to the proof of Proposition 6.2. Proposition 6.1 provides us isometries $\Phi_{n}: n^{-\frac{1}{p}}\left(\left(\mathbb{R}_{p}^{d}\right)_{p}^{n} / S_{n}\right) \rightarrow \mathscr{P}_{p}\left(\mathbb{R}_{p}^{d}\right)$. We denote by $I_{k}$ the image of $\Phi_{2^{k}}$. We have $I_{k} \subset I_{k+1}$ for every $k \in \mathbb{N}$. Since the union $\cup_{k=1}^{\infty} I_{k}$ is dense in $\mathscr{P}_{p}\left(\mathbb{R}_{p}^{d}\right)$ it follows that

$$
M_{2}\left(\mathscr{P}_{p}\left(\mathbb{R}_{p}^{d}\right)\right)=\sup _{k \in \mathbb{N}} M_{2}\left(I_{k}\right)=\sup _{k \in \mathbb{N}} M_{2}\left(\left(\mathbb{R}_{p}^{d}\right)_{p}^{k} / S_{k}\right)
$$

By Proposition 5.2 we have $M_{2}\left(\left(\mathbb{R}_{p}^{d}\right)_{p}^{k} / S_{k}\right) \leq M_{2}\left(\left(\mathbb{R}_{p}^{d}\right)_{p}^{k}\right)=M_{2}\left(\mathbb{R}_{p}^{d k}\right)$. Proposition 7.3 implies that $M_{2}\left(\mathbb{R}_{p}^{d k}\right) \leq 4 \sqrt{p-1}$. Hence, $M_{2}\left(\mathscr{P}_{p}\left(\mathbb{R}_{p}^{d}\right)\right) \leq$ $4 \sqrt{p-1}$.

The following example shows that Theorem[1(1,2) does not hold in general for infinitely sheeted coverings and infinite groups of isometries.

Example 7.4. Consider the $d$-dimensional Hamming cube $\Omega^{d}$, i.e a set $\{0,1\}^{d}$ with the $L_{1}$ metric. For the Markov type constants $M_{2}\left(\Omega^{d}\right)$ we have

$$
M_{2}\left(\Omega^{d}\right) \underset{d \rightarrow \infty}{\longrightarrow} \infty,
$$

see [9, Section 9.4].

The Hamming cube $\Omega^{d}$ can be converted to a metric graph $G\left(\Omega^{d}\right)$ by adding edges of length 1 between every two points $x, y \in \Omega^{d}: d(x, y)=1$. Consider the universal cover $\widetilde{G}\left(\Omega^{d}\right)$ of $G\left(\Omega^{d}\right)$. The graph $\widetilde{G}\left(\Omega^{d}\right)$ is a metric tree and consequently $M_{2}\left(\widetilde{G}\left(\Omega^{d}\right)\right) \leq 30$, see $[10]$.

Thus for a large enough $d$ we have $M_{2}\left(\widetilde{G}\left(\Omega^{d}\right)\right) \leq 30<M_{2}\left(G\left(\Omega^{d}\right)\right)$.

Definition 7.5. Let $X$ and $Y$ be metric spaces. A map $\chi: X \rightarrow Y$ is a submetry, iff for every $x \in X$ and every $r>0$

$$
\chi(B(x, r))=B(\chi(x), r),
$$

where $B(x, r)$ denotes the closed ball with center $x$ and radius $r$.

The following conjecture suggests a uniform approach to Propositions 4.2 and 5.2 .

Conjecture 7.6. Let $X$ and $Y$ be metric spaces such that there exists a submetry $\chi: X \rightarrow Y$, such that for every $y \in Y$ the set $\chi^{-1}(y)$ is finite. Then $M_{2}(X) \geq M_{2}(Y)$.

We did not found a proof, or a counter example to this conjecture. But we have an example which shows that our method, i.e., lifting of a Markov walks does not work, see Proposition 7.7 and Example 7.8 . 
Proposition 7.7. There exist finite metric spaces $\widetilde{X}, X$, a submetry $\chi: \widetilde{X} \rightarrow X$, a stationary reversible Markov chain $\left\{Z_{t}\right\}_{t=0}^{\infty}$ on a finite set $S$, and an injective map $f: S \rightarrow X$ such that $f\left(Z_{t}\right)$ does not admit a metric lift along $\chi$.

The proof of Proposition 7.7 occupies the rest of this section. The construction is given in the following example.

Example 7.8. Let $X=\left\{x_{1}, x_{2}, x_{3}, x_{4}\right\}, G_{X}$ a graph with vertex set $X$ and 5 edges connecting all pairs of vertices except $x_{2}$ and $x_{4}$. We consider $X$ as a metric space with metric induced from $G_{X}$, i.e distance between every pair of points except $\left\{x_{2}, x_{4}\right\}$ equals 1 . And distance between $x_{2}$ and $x_{4}$ equals 2.

Let $\left\{Z_{t}\right\}_{t=0}^{\infty}$ be a markov chain on the set $S=X=\left\{x_{1}, \ldots, x_{4}\right\}$ with stationary distribution $\left(\frac{3}{10}, \frac{2}{10}, \frac{3}{10}, \frac{2}{10}\right)$ and transition matrix $A=\left[\begin{array}{cccc}0 & \frac{1}{3} & \frac{1}{3} & \frac{1}{3} \\ \frac{1}{2} & 0 & \frac{1}{2} & 0 \\ \frac{1}{3} & \frac{1}{3} & 0 & \frac{1}{3} \\ \frac{1}{2} & 0 & \frac{1}{2} & 0\end{array}\right]$, and let $f=i d$.

Let $\widetilde{X}=\left\{\widetilde{x_{1}}, \ldots, \widetilde{x}_{12}\right\}, G_{\widetilde{X}}$ a graph with vertex set $X$ and 16 edges. The first group of edges forms the loop $\widetilde{x_{1}}, \ldots, \widetilde{x}_{12}$. The second group contains remaining 4 edges connecting following pairs of vertices $\left\{\widetilde{x}_{3}, \widetilde{x}_{1}\right\},\left\{\widetilde{x}_{3}, \widetilde{x}_{5}\right\}$, $\left\{\widetilde{x_{9}}, \widetilde{x}_{7}\right\},\left\{\widetilde{x}_{9}, \widetilde{x}_{11}\right\}$. Again we consider $\widetilde{X}$ as a metric space with metric induced from $G_{\tilde{X}}$.

Let $r_{4}: Z_{+} \rightarrow\{1,2,3,4\}$ be the reminder of a number modulo 4 . Let $\chi: \widetilde{X} \rightarrow X$ be a map defined by

$$
\chi\left(\widetilde{x}_{i}\right)=x_{r_{4}(i)} .
$$

Note that $\chi$ is a locally surjective graph homomorphism between $G_{\widetilde{X}}$ and $G_{X}$. Hence, $\chi$ is a submetry.

Lemma 7.9. Let $\left\{\widetilde{Z}_{t}\right\}_{t=0}^{\infty}$ and $\left\{Z_{t}\right\}_{t=0}^{\infty}$ be stationary reversible Markov chains on finite sets $\widetilde{S}$ and $S$. Suppose that $\widetilde{Z}$ is a lift of $Z$ along a map $\sigma: \widetilde{S} \rightarrow S$. Then we have $P^{\widetilde{Z}}\left(\widetilde{s}_{1}, \sigma^{-1}\left(s_{2}\right)\right)=P^{Z}\left(\sigma\left(\widetilde{s}_{1}\right),\left\{s_{2}\right\}\right)$, for every $\widetilde{s_{1}}=\widetilde{S}$ and every $s_{2} \in S$.

Proof. The proof is built around the following equality, which follows from Definition 2.1

$$
A^{\widetilde{Z}}\left(\sigma^{-1}\left(s_{2}\right), \sigma^{-1}\left(s_{1}\right), \sigma^{-1}\left(s_{2}\right)\right)=A^{Z}\left(s_{2}, s_{1}, s_{2}\right),
$$

where $s_{1}, s_{2} \in S$. 
Fix $s_{2} \in S$, expanding left and right sides of (7.1) we obtain

$$
\sum_{\widetilde{s}_{1} \in \sigma^{-1}\left(s_{1}\right)}\left(A^{\widetilde{Z}}\left(\sigma^{-1}\left(s_{2}\right),\left\{\widetilde{s}_{1}\right\}\right) P^{\widetilde{Z}}\left(\widetilde{s}_{1}, \sigma^{-1}\left(s_{2}\right)\right)\right)=A^{Z}\left(\left\{s_{2}\right\},\left\{s_{1}\right\}\right) P^{Z}\left(s_{1},\left\{s_{2}\right\}\right),
$$

Reversibility of Markov chains implies that $A^{Z}\left(\left\{s_{1}\right\},\left\{s_{2}\right\}\right)=A^{Z}\left(\left\{s_{2}\right\},\left\{s_{1}\right\}\right)$ and $A^{\widetilde{Z}}\left(\sigma^{-1}\left(s_{2}\right),\left\{\widetilde{s}_{1}\right\}\right)=A^{\widetilde{Z}}\left(\left\{\widetilde{s}_{1}\right\}, \sigma^{-1}\left(s_{2}\right)\right)$. Thus, we can rewrite (7.2) as

$$
\sum_{\widetilde{s}_{1} \in \sigma^{-1}\left(s_{1}\right)} \frac{A^{\widetilde{Z}}\left(\sigma^{-1}\left(s_{2}\right),\left\{\widetilde{s}_{1}\right\}\right)^{2}}{A^{\widetilde{Z}}\left(\left\{\widetilde{s}_{1}\right\}\right)}=\frac{A^{Z}\left(\left\{s_{2}\right\},\left\{s_{1}\right\}\right)^{2}}{A^{Z}\left(\left\{s_{1}\right\}\right)} .
$$

From Definition 2.1 we obtain

$$
\begin{gathered}
A^{Z}\left(\left\{s_{1}\right\}\right)=\sum_{\widetilde{s}_{1} \in \sigma^{-1}\left(s_{1}\right)} A^{\widetilde{Z}}\left(\left\{\widetilde{s}_{1}\right\}\right), \\
A^{Z}\left(\left\{s_{2}\right\},\left\{s_{1}\right\}\right)=\sum_{\widetilde{s}_{1} \in \sigma^{-1}\left(s_{1}\right)} A^{\widetilde{Z}}\left(\sigma^{-1}\left(s_{2}\right),\left\{\widetilde{s}_{1}\right\}\right) .
\end{gathered}
$$

Substituting the last two equalities into (7.3) and moving the denominator of the right side to the left we obtain

$$
\sum_{\widetilde{s}_{1} \in \sigma^{-1}\left(s_{1}\right)} A^{\widetilde{Z}}\left(\left\{\widetilde{s}_{1}\right\}\right) \sum_{\widetilde{s}_{1} \in \sigma^{-1}\left(s_{1}\right)} \frac{A^{\widetilde{Z}}\left(\sigma^{-1}\left(s_{2}\right),\left\{\widetilde{s}_{1}\right\}\right)^{2}}{A^{\widetilde{Z}}\left(\left\{\widetilde{s}_{1}\right\}\right)}=\left(\sum_{\widetilde{s}_{1} \in \sigma^{-1}\left(s_{1}\right)} A^{\widetilde{Z}}\left(\sigma^{-1}\left(s_{2}\right),\left\{\widetilde{s}_{1}\right\}\right)\right)^{2} .
$$

This is the equality case of the Cauchy-Schwarz inequality. Hence there exists a constant $\widetilde{C}=\widetilde{C}\left(s_{1}, s_{2}\right)$ such that

$$
\frac{A^{\widetilde{Z}}\left(\sigma^{-1}\left(s_{2}\right),\left\{\widetilde{s}_{1}\right\}\right)}{A^{\widetilde{Z}}\left(\left\{\widetilde{s}_{1}\right\}\right)} \stackrel{\text { def }}{=} P^{\widetilde{Z}}\left(\widetilde{s}_{1}, \sigma^{-1}\left(s_{2}\right)\right)=\widetilde{C},
$$

for every $\widetilde{s}_{1} \in \sigma^{-1}\left(s_{1}\right)$. From (7.4) and (7.5) it follows that

$$
\frac{A^{Z}\left(\left\{s_{2}\right\},\left\{s_{1}\right\}\right)}{A^{Z}\left(\left\{s_{1}\right\}\right)} \stackrel{\text { def }}{=} P^{Z}\left(s_{1},\left\{s_{2}\right\}\right)=\widetilde{C} .
$$

Lemma 7.10. Let $\left\{\widetilde{Z}_{t}\right\}_{t=0}^{\infty}$ and $\left\{Z_{t}\right\}_{t=0}^{\infty}$ be stationary reversible Markov chains on finite sets $\widetilde{S}$ and $S$. Suppose that $\widetilde{Z}$ is a lift of $Z$ (see Definition [2.5) along a map $\sigma: \widetilde{S} \rightarrow S$. Let $s_{1}, s_{2} \in S$ be such that $A^{Z}\left(\left\{s_{1}\right\},\left\{s_{2}\right\}\right) \neq 0$. Let $\widetilde{S}_{1} \subset \sigma^{-1}\left(s_{1}\right), \widetilde{S}_{2} \subset \sigma^{-1}\left(s_{2}\right)$ be such that

$$
\begin{aligned}
& A^{\widetilde{Z}}\left(\widetilde{S}_{1}, \sigma^{-1}\left(s_{2}\right) \backslash \widetilde{S}_{2}\right)=0, \\
& A^{\widetilde{Z}}\left(\widetilde{S}_{2}, \sigma^{-1}\left(s_{1}\right) \backslash \widetilde{S}_{1}\right)=0,
\end{aligned}
$$


Then

$$
\frac{A^{\widetilde{Z}}\left(\widetilde{S}_{1}\right)}{A^{Z}\left(\left\{s_{1}\right\}\right)}=\frac{A^{Z}\left(\widetilde{S}_{2}\right)}{A^{Z}\left(\left\{s_{2}\right\}\right)} .
$$

Proof. Let $\widetilde{s}_{1} \in \widetilde{S}_{1}$, Lemma 7.9 implies that

$$
P^{\widetilde{Z}}\left(\widetilde{s}_{1}, \sigma^{-1}\left(s_{2}\right)\right)=P^{Z}\left(s_{1},\left\{s_{2}\right\}\right),
$$

Using the assumption (7.7) we can rewrite this equality as

$$
A^{\widetilde{Z}}\left(\left\{\widetilde{s}_{1}\right\}, \widetilde{S}_{2}\right)=A^{\widetilde{Z}}\left(\widetilde{s}_{1}\right) \frac{A^{Z}\left(\left\{s_{1}\right\},\left\{s_{2}\right\}\right)}{A^{Z}\left(\left\{s_{1}\right\}\right)},
$$

Summing the previous equalities for all $\widetilde{s_{1}} \in \widetilde{S}_{1}$ we have

$$
A^{\widetilde{Z}}\left(\widetilde{S}_{1}, \widetilde{S}_{2}\right)=A^{\widetilde{Z}}\left(\widetilde{S}_{1}\right) \frac{A^{Z}\left(\left\{s_{1}\right\},\left\{s_{2}\right\}\right)}{A^{Z}\left(\left\{s_{1}\right\}\right)},
$$

The same argument shows that

$$
A^{\widetilde{Z}}\left(\widetilde{S}_{2}, \widetilde{S}_{1}\right)=A^{\widetilde{Z}}\left(\widetilde{S}_{2}\right) \frac{A^{Z}\left(\left\{s_{2}\right\},\left\{s_{1}\right\}\right)}{A^{Z}\left(\left\{s_{2}\right\}\right)},
$$

Since $A^{Z}\left(\left\{s_{1}\right\},\left\{s_{2}\right\}\right) \neq 0$ we obtain

$$
\frac{A^{\widetilde{Z}}\left(\widetilde{S}_{1}\right)}{A^{Z}\left(\left\{s_{1}\right\}\right)}=\frac{A^{\widetilde{Z}}\left(\widetilde{S}_{2}\right)}{A^{Z}\left(\left\{s_{2}\right\}\right)} .
$$

Proof of Proposition 7.7. Let $X, \widetilde{X}$ and $\chi$ be as in Example 7.8. By contradiction, suppose there exist $\left\{\widetilde{Z}_{t}\right\}_{t=0}^{\infty}$ a stationary reversible Markov chains on finite set $\widetilde{S}$ and a map $\widetilde{f}: \widetilde{S} \rightarrow \widetilde{X}$, such that Markov walk $\widetilde{f}\left(\widetilde{Z}_{t}\right)$ is a metric lift of $f\left(Z_{t}\right)$ along $\chi$. Note that since $f$ is injective the Markov chain $\widetilde{Z}_{t}$ is a lift of a Markov chain $Z_{t}$ along a map $\sigma: \widetilde{S} \rightarrow S$ defined by $\sigma=f^{-1} \circ \chi \circ \widetilde{f}$. For $1 \leq i \leq 4,1 \leq j \leq 12$ we denote $A^{Z}\left(x_{i}\right)$ by $p_{i}$ and $A^{\widetilde{Z}}\left(\widetilde{f}^{-1}\left(\widetilde{x}_{j}\right)\right)$ by $q_{j}$.

Let $i=1, \ldots, 11$, consider $\widetilde{x}_{i}$ and $\widetilde{x}_{i+1}$. By Lemma 7.10 applied to $s_{1}=$ $x_{r_{4}(i)}, s_{2}=x_{r_{4}(i+1)}, \widetilde{S}_{1}=\widetilde{f}^{-1}\left(\widetilde{x}_{i}\right), \widetilde{S}_{2}=\widetilde{f}^{-1}\left(\widetilde{x}_{i+1}\right)$ we have

$$
\frac{q_{i}}{p_{r_{4}(i)}}=\frac{q_{i+1}}{p_{r_{4}(i+1)}}
$$

These equalities imply that

$$
q_{3}=q_{1}=q_{5} \neq 0 \text {. }
$$

Lemma 7.10 applied to $s_{1}=x_{3}, s_{2}=x_{1}, \widetilde{S}_{1}=\widetilde{f}^{-1}\left(\widetilde{x}_{3}\right), \widetilde{S}_{2}=\widetilde{f}^{-1}\left(\left\{\widetilde{x}_{1}, \widetilde{x}_{5}\right\}\right)$ implies that

$$
\frac{q_{3}}{p_{3}}=\frac{q_{1}+q_{5}}{p_{1}}
$$


Since $p_{3}=p_{1}=\frac{3}{10}$ we have

$$
q_{3}=q_{1}+q_{5}
$$

This contradicts (7.10).

\section{REFERENCES}

[1] A. Andoni, A. Naor, and O. Neiman. Snowflake universality of Wasserstein spaces. ArXiv e-prints, September 2015.

[2] K. Ball. Markov chains, Riesz transforms and Lipschitz maps. Geometric and Functional Analysis GAFA, 2(2):137-172, 1992.

[3] Yair Bartal, Nathan Linial, Manor Mendel, and Assaf Naor. On Metric Ramsey-type Phenomena. In Proceedings of the Thirty-fifth Annual ACM Symposium on Theory of Computing, STOC '03, pages 463-472, New York, NY, USA, 2003. ACM.

[4] Ludwig Bieberbach. Über die Bewegungsgruppen der Euklidischen Räume. Mathematische Annalen, 70(3):297-336, 1911.

[5] Ludwig Bieberbach. Über die Bewegungsgruppen der Euklidischen Räume (Zweite Abhandlung.) Die Gruppen mit einem endlichen Fundamentalbereich. Mathematische Annalen, 72(3):400-412, 1912.

[6] Yu Burago, M Gromov, and G Perel'man. A.D. Alexandrov spaces with curvature bounded below. Russian Mathematical Surveys, 47(2):1, 1992.

[7] N. Linial, A. Magen, and A. Naor. Girth and Euclidean distortion. Geometric and Functional Analysis GAFA, 12(2):380-394, 2002.

[8] Manor Mendel and Assaf Naor. Spectral calculus and lipschitz extension for barycentric metric spaces. Analysis and Geometry in Metric Spaces, 1:163-199.

[9] Assaf Naor. An introduction to the Ribe program. Japanese Journal of Mathematics, $7(2): 167-233,2012$.

[10] Assaf Naor, Yuval Peres, Oded Schramm, and Scott Sheffield. Markov chains in smooth Banach spaces and Gromov-hyperbolic metric spaces. Duke Math. J., 134(1):165-197, 072006.

[11] John Nash. C ${ }^{1}$ Isometric Imbeddings. Annals of Mathematics, 60(3):383-396, 1954.

[12] Shin-Ichi Ohta. Markov Type of Alexandrov Spaces of Non-Negative Curvature. Mathematika, 55:177-189, 122009.

[13] Shin-Ichi Ohta and Mikaël Pichot. A note on Markov type constants. Archiv der Mathematik, 92(1):80-88, 2009.

[14] Karl-Theodor Sturm. On the geometry of metric measure spaces. Acta Mathematica, 196(1):65-131, 2006.

[15] Cédric Villani. Topics in optimal transportation. Number 58. American Mathematical Soc., 2003.

(Vladimir Zolotov) Steklov Institute of Mathematics, Russian Academy of Sciences, 27 Fontanka, 191023 St.Petersburg, Russia and Mathematics and Mechanics Faculty, St. Petersburg State University, Universitetsky Pr., 28, Stary Peterhof, 198504, Russia.

E-mail address, Vladimir Zolotov: paranuel@mail.ru 